Available online at:

https://acta-acustica.edpsciences.org

\title{
Generation of uncertainty envelopes for thin acoustic screens with uncertain parameters
}

\author{
Mathieu Gaborit ${ }^{1,2, *}$, Olivier Dazel ${ }^{1}$, Peter Göransson ${ }^{2}$, and Luc Jaouen ${ }^{3}$ \\ ${ }^{1}$ LAUM UMR CNRS 6613, Le Mans Université, 72085 Le Mans Cedex, France \\ ${ }^{2}$ MWL, KTH Royal Institute of Technology, 10044 Stockholm, Sweden \\ ${ }^{3}$ Matelys Research Laboratory, 69120 Vaulx-en-Velin, France
}

Received 26 January 2020, Accepted 25 May 2020

\begin{abstract}
The properties of the materials used for building sound proofing systems are known to exhibit large variations. These may lead to significant differences in the acoustic responses within a given material batch, particularly when resistive screens are used as a surface component for a multi-layered absorbing panel. In such thin films, it is mostly the thickness and the flow resistivity, but in some cases also the porosity, that are difficult to control in the production process. All these potential variations influence the acoustic response of the complete panel. In the present contribution, a method to isolate and evaluate the effect of uncertainties in a film is proposed. Using a transmission line approach, it is shown to be possible to predict the modification of the response induced by the uncertainties. The proposed technique is then adapted to determine uncertainty envelopes of the absorption coefficient, for experimentally acquired responses, that are closer to measured envelopes as compared to those generated using Monte Carlo simulations or simplified approaches. The method is tested both on numerical and experimental cases and shows, in both cases, a very good agreement with the reference solutions. Unlike Monte Carlo approaches, the proposed method does not require a massive computational effort which makes it suitable for real life applications.
\end{abstract}

Keywords: Acoustics screens, Uncertainties, Thin layers, Poroelastic media

\section{Introduction}

Reducing noise through dedicated acoustic treatments is essential in modern society to counteract the negative impact on health that is linked to exposure. Many approaches have been proposed over the years, either with a broadband noise reduction objective [1] or targeting a given frequency band [2-4]. Recent reports and directives [5-7] set evermore ambitious targets in term of noise mitigation for the future. This overall endeavour to reduce noise and its impact requires the development of efficient, versatile strategies to be combined with punctual, more specific solutions to mitigate particular features of the noise content (emerging tones for instance).

A widely used and rather inexpensive approach to noise reduction over a fairly large frequency band uses poroelastic materials (foam, fibre panels, etc.) arranged as a multi-layered laminate. These composite structures usually involve a small number of foam/fibrous layers with carefully chosen properties and thicknesses in order to meet specific absorption objectives [1]. To protect the panel and sometimes fine tune its properties, thin films can be added on

*Corresponding author: gaborit@kth.se the free surface $[8,9]$. Because of the films' airflow resistivity is often high they are known to play a prominent role in the behaviour of the overall systems [10-12]. As their precise characterisation might be challenging [13], it is of primary importance to understand how the uncertainties on the film parameters induce variations of the overall system response. Apart from this objective, another goal of the study is to propose an efficient method to compute the associated response frequency envelope curves both on simulated response and on experimental results. It is shown that the proposed approach allows one to merge statistical data from the characterisation of the screen with a single measurement of the overall system response. The resulting envelopes are shown to convey the same statistical information as Monte Carlo results obtained by measuring a large number of samples.

The paper is organised as follows. In the first section a study model is introduced and used to derive the influence of the film and its uncertain parameters on the response. A technique for combining an existing deterministic response of a panel and statistical information on the film to infer the associated envelope is discussed. In a second section, the proposed developments are tested against Monte Carlo simulations and validated. A third section presents an experimental set-up to measure the absorption coefficient 
of different laminates. Using a large number of samples from two different films, a statistical characterisation is performed following a procedure described in the literature [13]. Using the same rig, different systems composed of an absorbing backing lined by one of these films are measured for an important number of film samples in order to obtain experimental envelopes. The experimental results are compared to those given by the proposed envelope generation technique. A final section discusses the results.

\section{Theoretical background}

\subsection{Position of the problem}

The key idea of this section is to write the system response such that it is possible to isolate the contribution of uncertain film parameters. The chosen configuration is presented in Figure 1 and involves a backing represented by its surface impedance $Z_{B}$, a resistive but permeable layer (film, screen, etc.) of thickness $d$ and the so-called incident medium in which the excitation propagates.

In the following, a positive time convention $e^{j \omega t}$ is assumed where $\omega$ is the pulsation, $t$ is the time variable and $j^{2}=-1$.

\subsection{Transmission through the film}

Throughout this work, the film layer is assumed to be thin, of the order of half a millimetre. It is furthermore assumed that the elastic deformation of the film may be neglected. This allows for a class of simplified models where the film may be regarded as an equivalent fluid $[1,14]$. In the examples, a limp model was used for this purpose.

Based on the above assumptions, the thin film layer is modelled through an equivalent characteristic impedance:

$$
Z_{f}=\sqrt{\rho_{f} K_{f}}
$$

where $\rho_{f}$ and $K_{f}$ are respectively the equivalent density and dynamic bulk modulus. Different expressions of these quantities exist in the literature $[1,15,16]$ but are out of the scope of this paper and will not be discussed in the present contribution.

\subsubsection{Evaluating the surface impedance}

In order to determine the response of the multi-layered system to a plane wave excitation, one needs its surface impedance $Z_{s}$. Using an equivalent fluid model for the film, this quantity can be deduced from the backing surface impedance $Z_{B}$ and the film properties using the impedance translation formula [1]:

$$
Z_{s}=Z_{f} \frac{Z_{B}+j Z_{f} \tan \left(k_{f} d\right)}{Z_{f}+j Z_{B} \tan \left(k_{f} d\right)}
$$

where,

$$
k_{f}=\omega \cos \theta \sqrt{\rho_{f} / K_{f}},
$$

is the wavenumber in the film.

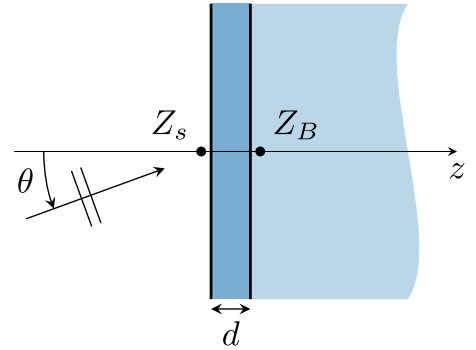

Figure 1. Configuration under study. The backing is known through its surface impedance $Z_{B}$, the system is impinged by a plane wave propagating at an angle $\theta$ from the normal to the surface.

As the thickness of the film is small compared to the wavelength $\left(\varepsilon=k_{f} d \ll 1\right)$, a first order Taylor expansion of the tangent gives $\tan \left(k_{f} d\right)=k_{f} d+\mathcal{O}\left(k_{f} d\right)$. After substitution in (2) and simplification, this may be rewritten as:

$$
Z_{s} \approx \frac{Z_{B}+j Z_{f} k_{f} d}{1+j Z_{B} Z_{f}^{-1} k_{f} d},
$$

which may be simplified further, through a Taylor expansion of the denominator:

$$
Z_{s}=Z_{B}-j Z_{B}^{2} Z_{f}^{-1} k_{f} d+j Z_{f} k_{f} d+\mathcal{O}\left(\left(k_{f} d\right)^{2}\right) .
$$

Finally, substituting equations (3) and (1), and neglecting the $\left(k_{f} d\right)^{2}$ term gives:

$$
Z_{s}=Z_{B}+\underbrace{j \omega d \cos \theta\left(\rho_{f}-Z_{B}^{2} K_{f}^{-1}\right)}_{Z_{F}} .
$$

The influence of the film is then entirely captured in $Z_{F}$ which, apart from the angle of incidence and the thickness of the screen, shows a dependency on the backing impedance $Z_{B}$.

\subsubsection{Introducing uncertainties}

As indicated in the introduction, the parameters of acoustic screens are seldom known with perfect accuracy. The sources of uncertainty can be either linked to samplewise variations or to the characterisation itself. These films, woven or non-woven, are rather delicate and it is difficult to ensure that cutting and manipulating the samples does not alter their properties. Thus, different factors tend to render these media challenging to characterise even though standardised procedures are available $[13,17]$. Their influence on the acoustic performance is mainly controlled by two properties, the airflow resistivity $\sigma$ and the thickness $d[1,12]$. In this section, the corresponding uncertainties induced on the model parameters of the medium are considered in terms of the higher level descriptors: $\rho_{f}$ and $K_{f}$. Through these two descriptors, and the thickness $d$, the resulting deviation may be directly introduced into (6). It should be noted that the hypotheses leading to (6) henceforth are assumed to apply. 
In order to determine the variation of $Z_{F}$ when the different parameters vary, the total differential of $Z_{F}$ is obtained as:

$$
\Delta Z_{F}=j \omega \cos \theta\left[\left(\rho_{f}-Z_{B}^{2} K_{f}^{-1}\right) \Delta d+d \Delta \rho_{f}+d Z_{B}^{2} K^{-2} \Delta K_{f}\right] .
$$

Using this, the variation of $Z_{s}$ as a whole is then calculated through:

$$
Z_{s}=Z_{B}+Z_{F}+\Delta Z_{F} .
$$

Thus, in the case of a variation of $\rho_{f}$ alone, the resulting surface impedance is given by:

$$
Z_{s}=Z_{B}+Z_{F}+j \omega d \cos \theta d \Delta \rho_{f},
$$

which is independent of the backing system. In other cases, e.g. when the thickness or the bulk modulus are known to vary, the surface impedance of the backing system is required at the frequency of interest. The cases for which this last quantity is not readily available are discussed in Section 2.3.

\subsubsection{Deriving reflection and absorption coefficients}

Considering the deviation of the surface impedance $Z_{s}=\bar{Z}_{s}+\Delta Z_{s}$, it is straightforward to compute the reflection coefficient:

$$
R=\frac{\bar{Z}_{s}+\Delta Z_{s}-Z_{0}}{\bar{Z}_{s}+\Delta Z_{s}+Z_{0}}
$$

and factorising $\bar{Z}_{s}+Z_{0}$, the denominator contains a small parameter $\Delta Z_{s}\left(\bar{Z}_{s}+Z_{0}\right)^{-1}$ and thus may be expanded in a Taylor series, which allows to separate the nominal and deviated part of $R$ as,

$$
R=\underbrace{\frac{\bar{Z}_{s}-Z_{0}}{\bar{Z}_{s}+Z_{0}}}_{\bar{R}}(1+\underbrace{\frac{2 Z_{0} \Delta Z_{s}}{\bar{Z}_{s}^{2}-Z_{0}^{2}}}_{\eta})+\mathcal{O}\left(\frac{\Delta Z_{s}^{2}}{\bar{Z}_{s}^{2}-Z_{0}^{2}}\right) .
$$

From (11), one can derive the absorption coefficient as (with $\mathcal{R}(\eta)$ denoting the real part of $\eta$ ):

$$
\alpha=1-|\bar{R}|^{2}|1+\eta|^{2}=\underbrace{1-|R|^{2}}_{\bar{\alpha}}-\underbrace{|R|^{2}\left(2 \mathcal{R}(\eta)+|\eta|^{2}\right)}_{\Delta \alpha} .
$$

\subsection{Envelope determination}

Having established equation (7), it is now possible to generate an envelope curve using as input a single response of the overall system and information on the uncertainties in the film. This process will be outlined in the following, based on the notation introduced in Figure 2. Here two possibly independent sets of measurements are used. On one hand, a characterisation of the film is required including a rather accurate estimation of how properties are statistically

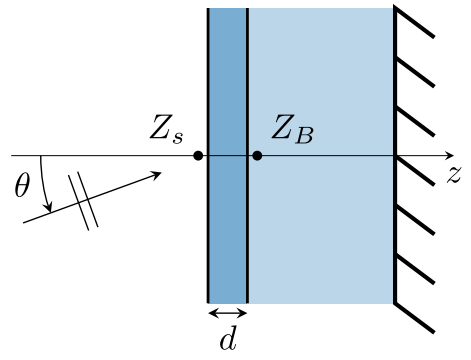

Figure 2. System considered for the addition of the envelopes on a precomputed response.

distributed, for which different techniques exist, either based on quasi-static or dynamic measurements $[13,17]$. On the other hand, a deterministic response of the overall system is needed which may be readily measured using e.g. standard impedance tube techniques [18].

Depending on which parameters of the equivalent fluid model are affected, and thus on the type of uncertainty considered, the envelope generation process follows slightly different routes, essentially following Section 2.2.2. In cases when the uncertainty on $\rho_{f}$ is evaluated, the overall surface impedance and its computed deviation are independent. On the other hand, if either $d$ or $K_{f}$ are affected by uncertainties, generating the frequency envelope requires the computation of $Z_{B}$ and thus the overall surface impedance $Z_{s}$ needs to be known. To obtain $Z_{B}$, the impedance translation theorem is used anew, and the value of the overall surface impedance $Z_{S}$ has to be obtained implying a translation through the film thus its parameters has to be known. These may be obtained by averaging the corresponding experimentally characterised values, in order to obtain the average value of the impedance $\bar{Z}_{B}$. Once $\bar{Z}_{B}$ is entirely determined, there only remains the computation of the deviation to the mean response from equation (7).

\section{Numerical validation}

As a validation of the proposed approach, numerical simulations are employed. In the current work, envelopes generated using the previously presented steps are compared to results obtained from a Monte Carlo based analysis.

For the numerical validation, three different configurations are used (see Fig. 3). In order to assess the limitations of the proposed method in relation to thin films of different design and manufacturing processes, a woven and a nonwoven film are investigated. The physical parameters of the media are taken from the literature $[1,11,13]$ and gathered in Table 1 for reference. The following properties are used for air: $\rho_{0}=1.213 \mathrm{~kg} \cdot \mathrm{m}^{-3}, \operatorname{Pr}=0.71, \eta_{0}=1.839 \times$ $10^{-5} \mathrm{~Pa} \cdot \mathrm{s}, P_{0}=1.01325 \times 10^{5} \mathrm{~Pa}$, and $\gamma_{0}=1.4$. Note that in the following, a Johnson-Champoux-Allard (JCA) model $[15,16]$ is used to determine the equivalent fluid quantities for the surface layer and only the corresponding parameters are used.

The two different films attached to a slab of foam and impinged by a plane wave at an angle $\theta=0^{\circ}$ or $\theta=60^{\circ}$, 
a)

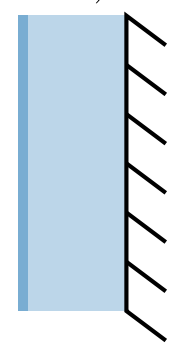

b)

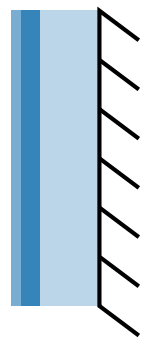

c)

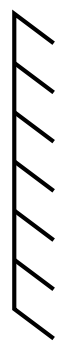

Figure 3. Test systems considered. (a) The film is placed on a single layer backing. Two configurations are tested: foam backing ( $L=50 \mathrm{~mm}$ ). (b) The film is set on a two-layers backing (thickness of the backing layers is respectively $10 \mathrm{~mm}$ and $30 \mathrm{~mm}$ ). (c) An air plenum $(L=200 \mathrm{~mm})$ if formed behind the film. The properties of the media used for these tests are reported in Table 1 .

Table 1. Properties of media used in simulated tests, taken from the literature and especially [1, 11, 13].

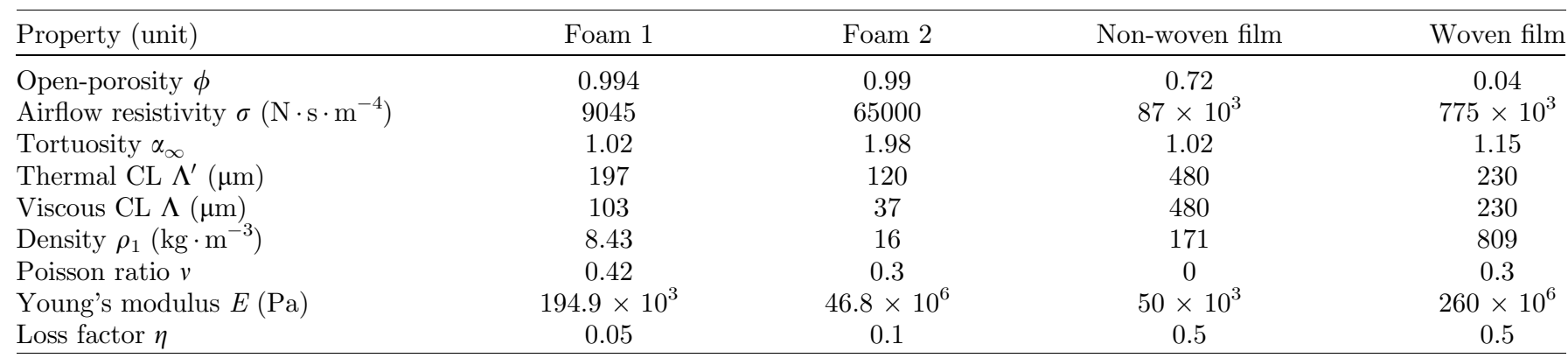

CL stands for characteristic length.

give the results shown in Figure 4. These angles were selected in order to illustrate the resulting behaviour in both normal and oblique incidence. In this figure, the results from the proposed approach are compared with the Monte Carlo (MC) generated reference solutions. The MC references are generated by computing the response of the system corresponding to 300 samples of the varying parameter $\xi$ drawn from a normal distribution $\mathcal{N}(\bar{\xi}, \Delta \xi)$ with $\Delta \xi=0.1 \bar{\xi}$. Statistical indicators are then used to deduce the reference curves (standard deviation and extremal values for each frequency). The solutions from the proposed approach are generated for different deviations $n \Delta \xi$ with $n=1,2,3$ in order to reach different confidence levels.

Two different representations are proposed: the response envelope, plotted as a regular absorption coefficient and the centred envelope where the mean curve is subtracted from the envelope curves. This second representation (shown on the four bottom plots of Fig. 4) is especially useful when the comparison involves the generated envelope amplitudes over the entire frequency range under consideration.

Several points are worth noting about the results presented in Figure 4. The good agreement between the $n=1$ envelopes predicted by the proposed approach and the MC standard deviation envelopes could perhaps have been anticipated given that this envelope correspond to a $68 \%$ confidence interval. Similarly, almost all the realisations are inside the $n=2$ envelope (corresponding to a $95 \%$ confidence level). Over the considered frequency range, no realisation is completely outside the predicted envelopes) but some are partially outside of the envelope corresponding to a $99.7 \%$ confidence level $(n=3)$.

One must keep in mind that the differences between the predicted and empirical envelopes are of very low amplitudes and that a slight change in the model could cause such effects. It is also important to remember that the proposed approach is based on several approximations supported by the fact that the film is thin which might impair the overall accuracy. Furthermore, it is important to note that no major difference in accuracy is observed when increasing the angle of incidence $\theta$.

In order to demonstrate that the proposed approach works for different backings, two more cases are studied. Figure 5 shows the results obtained for a facing placed upstream a $200 \mathrm{~mm}$ air plenum which correspond to configuration in Figure 3c. This case triggers a large number of resonances but the predicted envelopes are very similar to the empirical ones. It is interesting to note, from a modelling point of view, that the envelopes constrict near resonances and expand in a very repeatable pattern. This feature was also visible in Figure 4 to a certain extent even though the resonances are not as sharp. An interesting phenomenon is observed on the $\theta=0^{\circ}$ responses of Figure 4: constrictions of the envelope occur even though no resonance is visible for the absorption coefficient.

As a last validation test, Figure 6 shows the response and envelopes when the film facings are placed onto two 

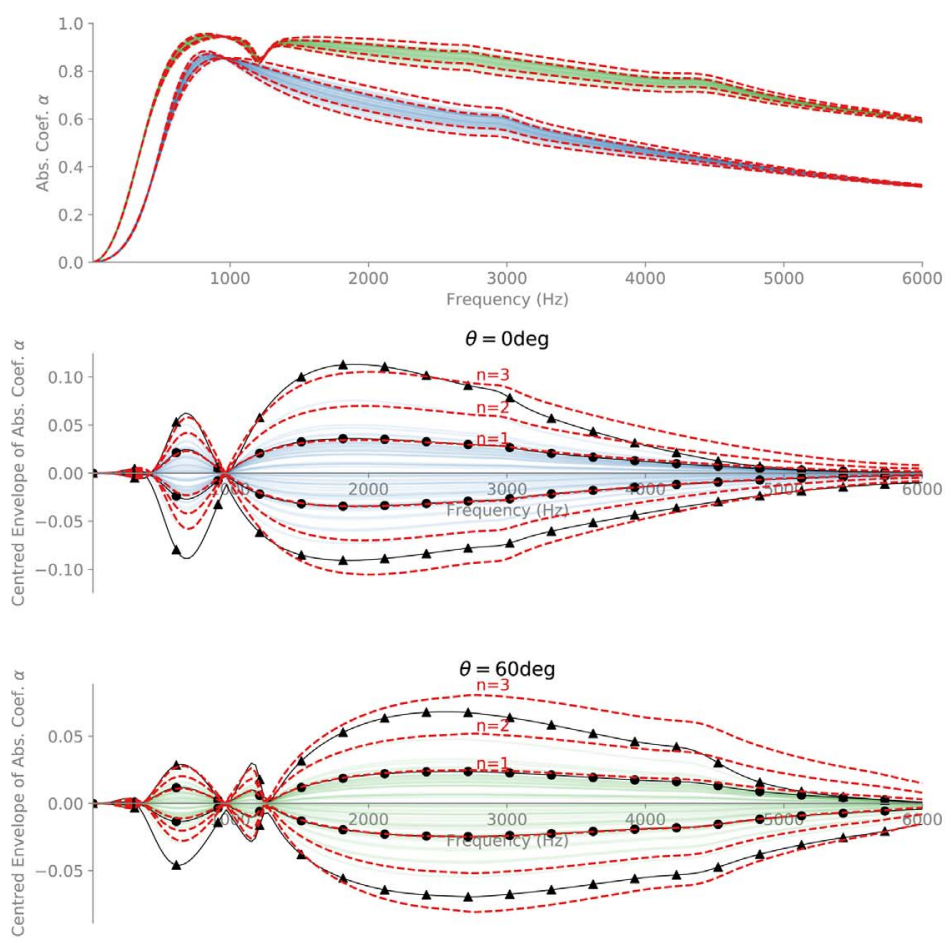

(a) Non-woven film
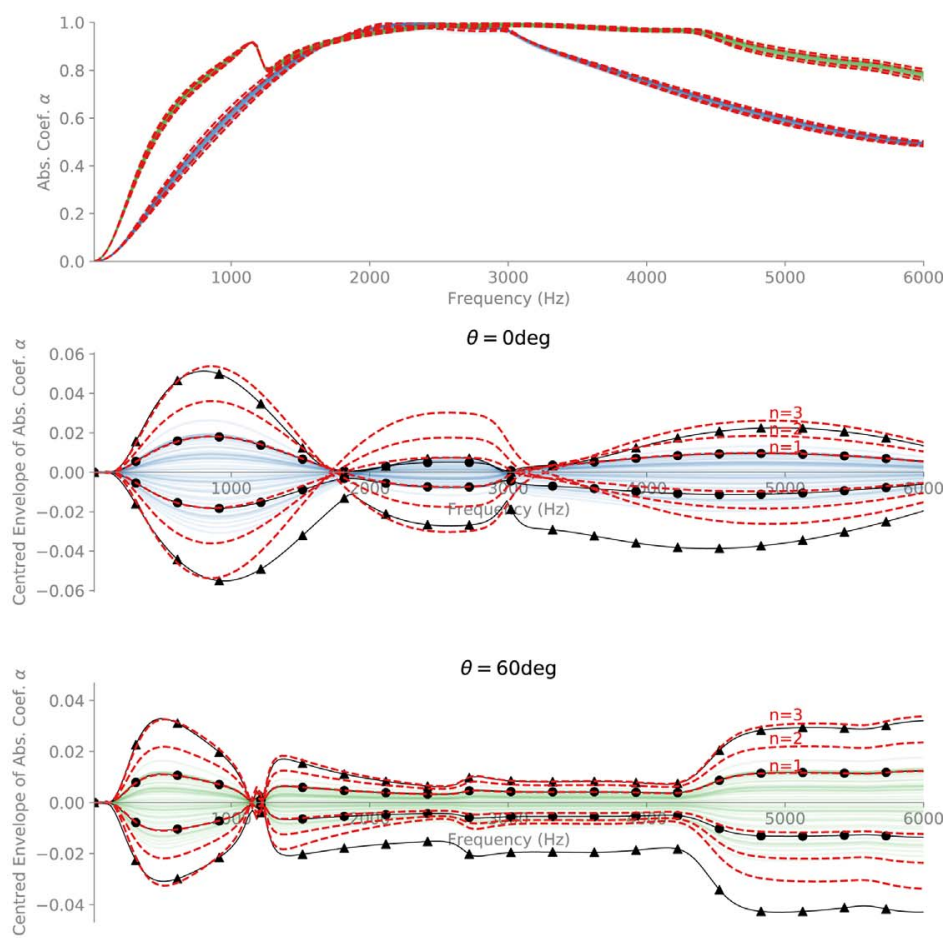

(b) Woven film

Figure 4. Case of a varying thin film placed over a poro-elastic slab with a rigid backing. Comparison between Monte Carlo envelopes (realisations in blue/green, realisation-wise standard deviation envelope with bullets • and extremal envelopes with triangles $\mathbf{\Delta}$ ) and envelope from the proposed method (dashed red, $n$ being the multiplier to the standard deviation of the varying parameter). Absorption coefficient (top most axis) and centred envelopes (two lower axes) for two angles of incidence $\left(0^{\circ}\right.$ in blue and $60^{\circ}$ in green). 

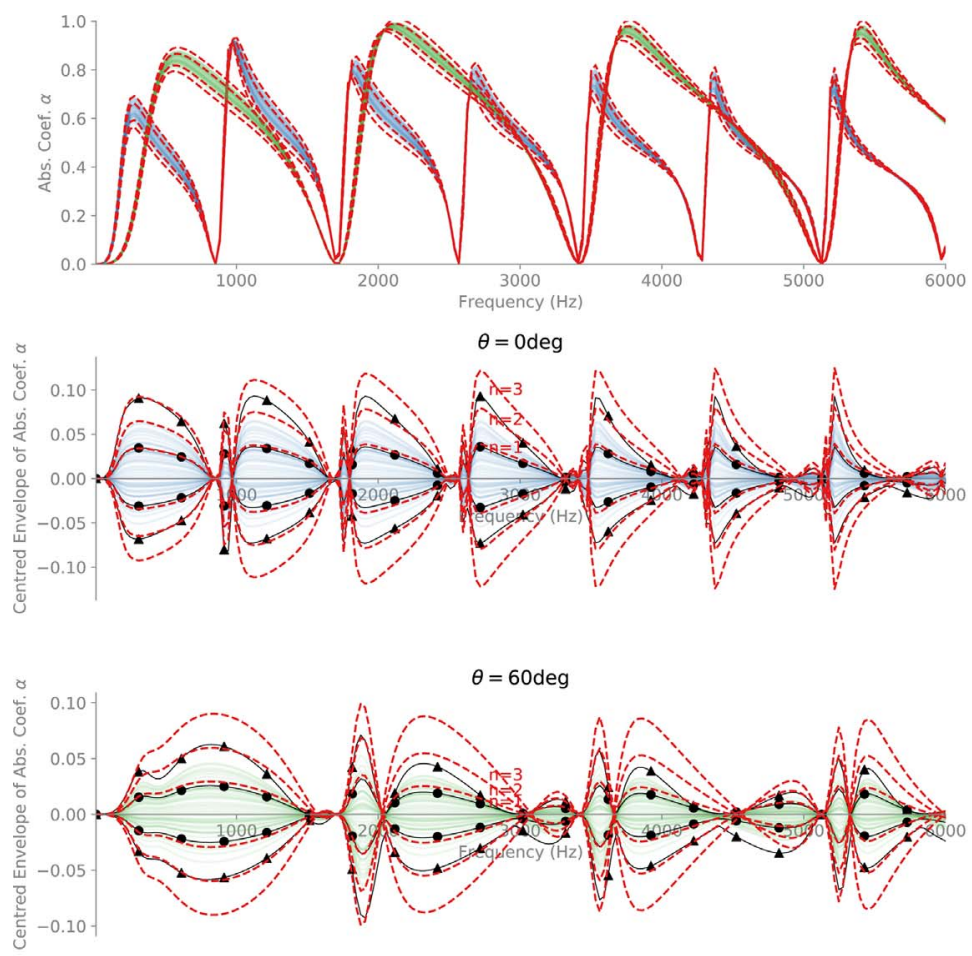

(a) Non-woven film
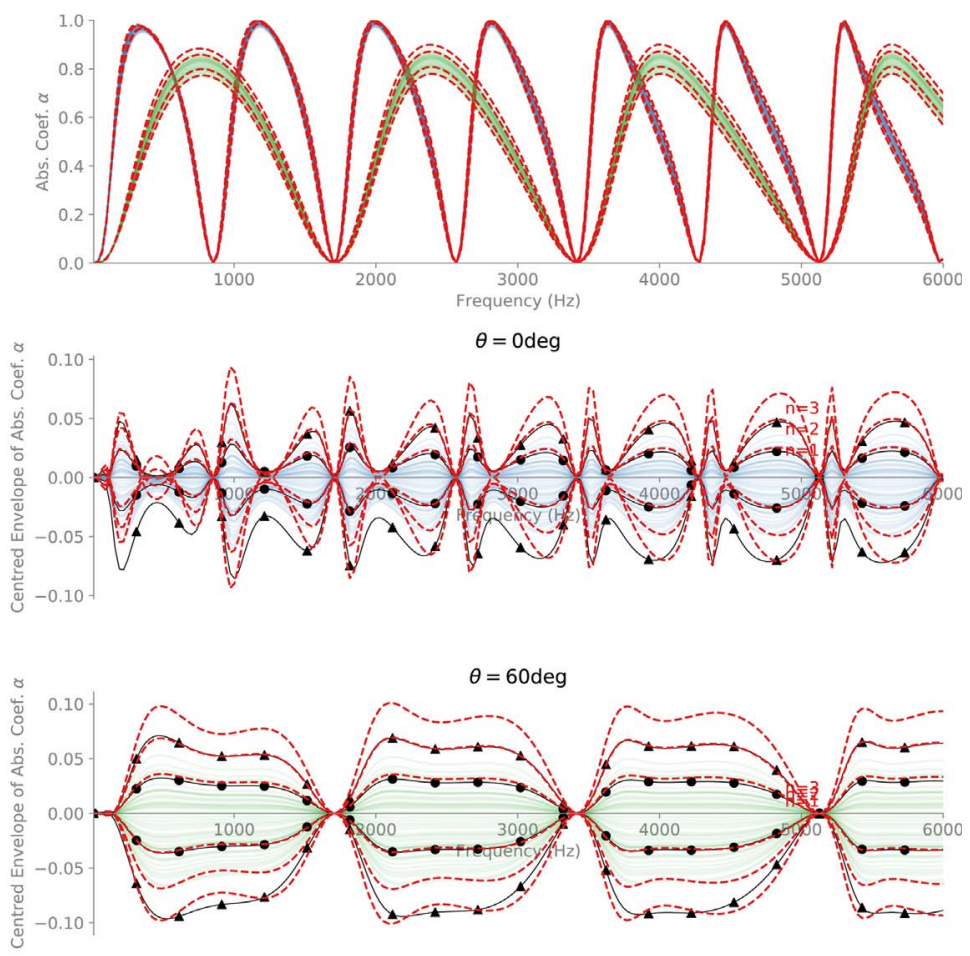

(b) Woven film

Figure 5. Case of a varying thin film upstream a $200 \mathrm{~mm}$ air plenum. Quantities and line styles identical to Figure 4. 

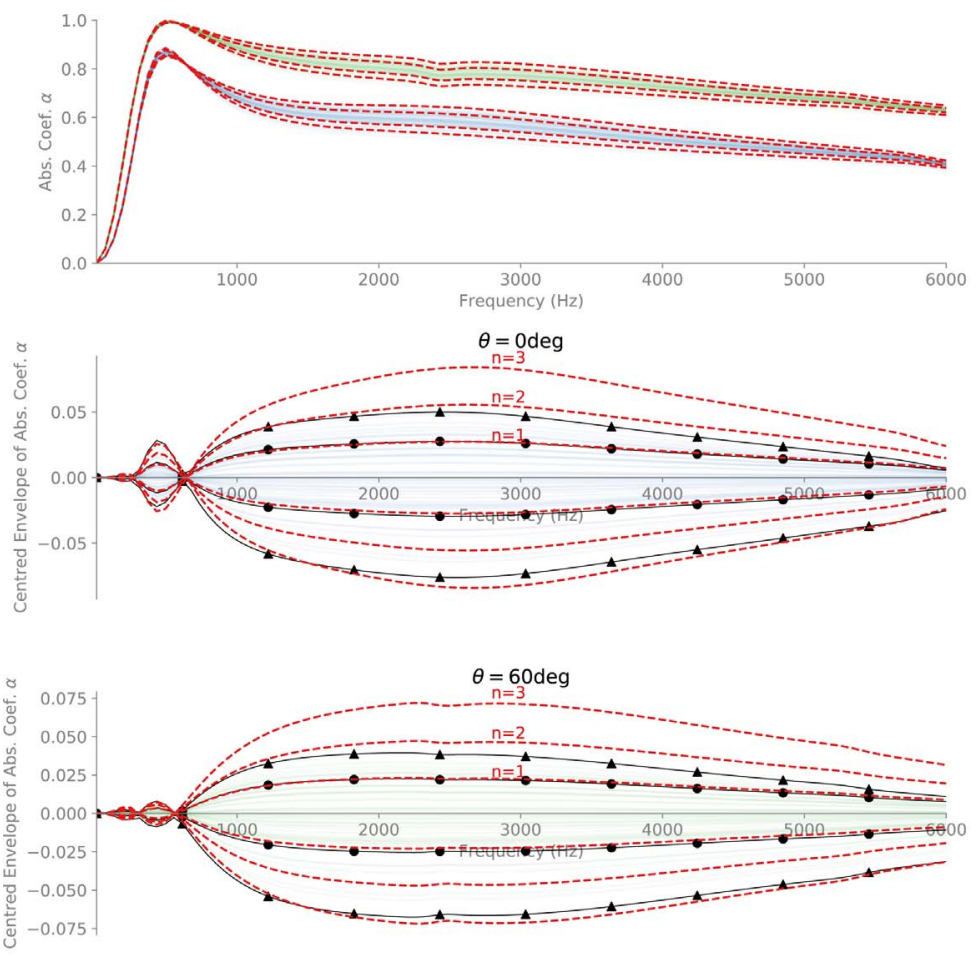

(a) Non-woven film
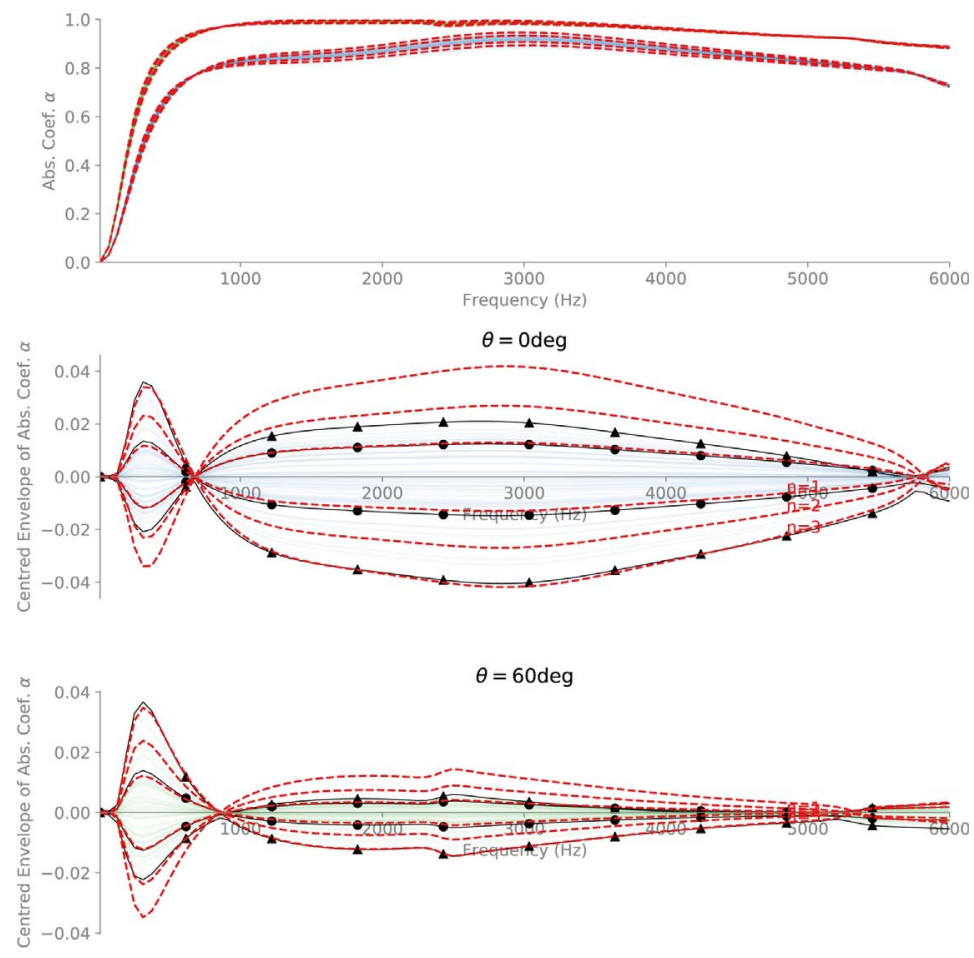

(b) Woven film

Figure 6. Case of a varying thin film placed over two layers of poro-elastic media of thickness $10 \mathrm{~mm}$ and $30 \mathrm{~mm}$. Quantities and line styles identical to Figure 4. 


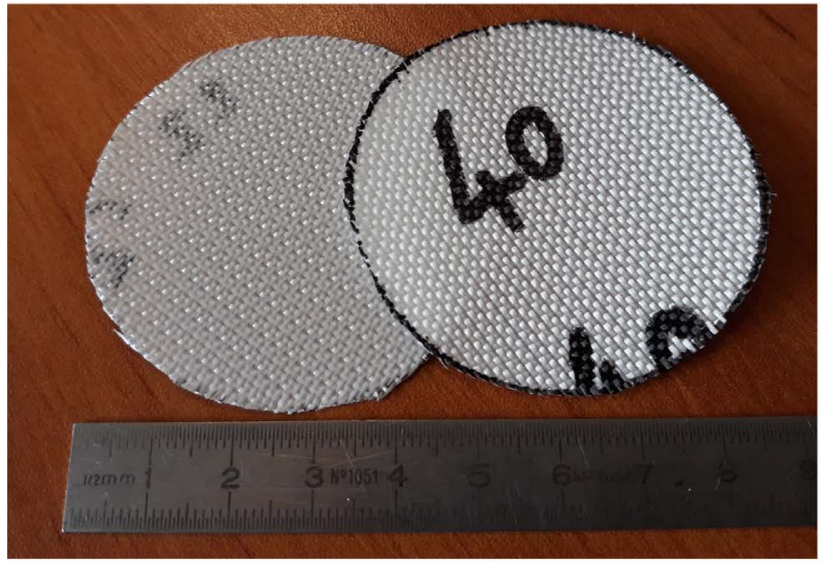

(a) Woven facing

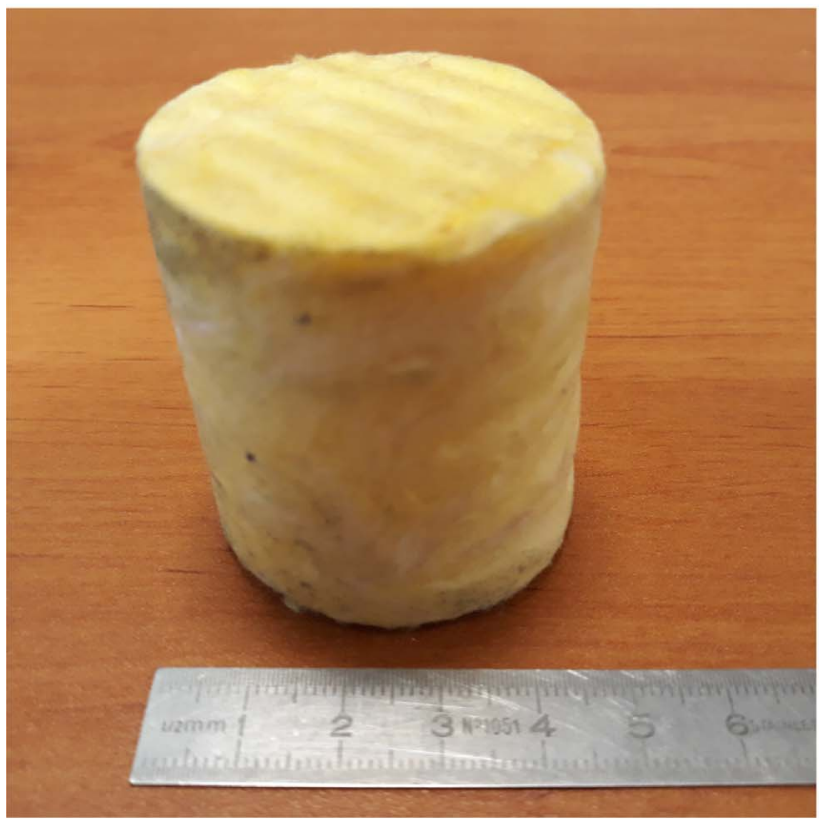

(c) Glass wool backing

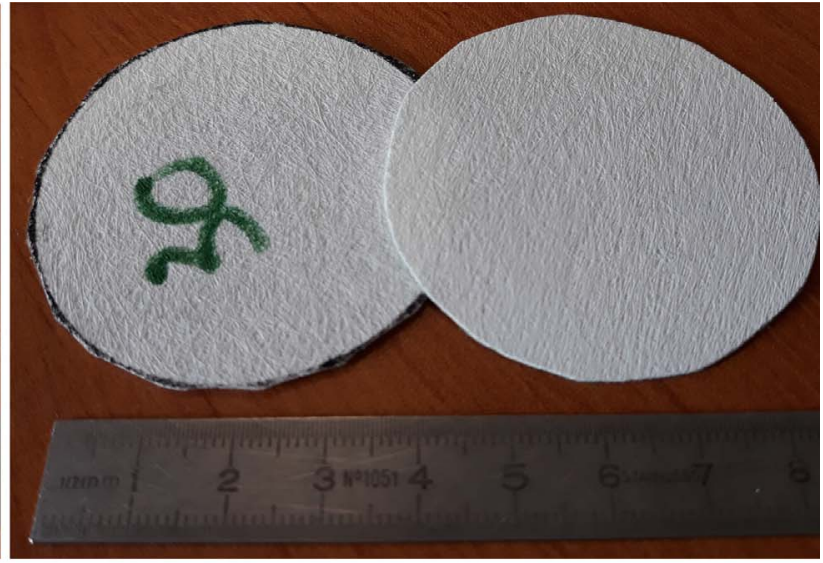

(b) Non-woven facing

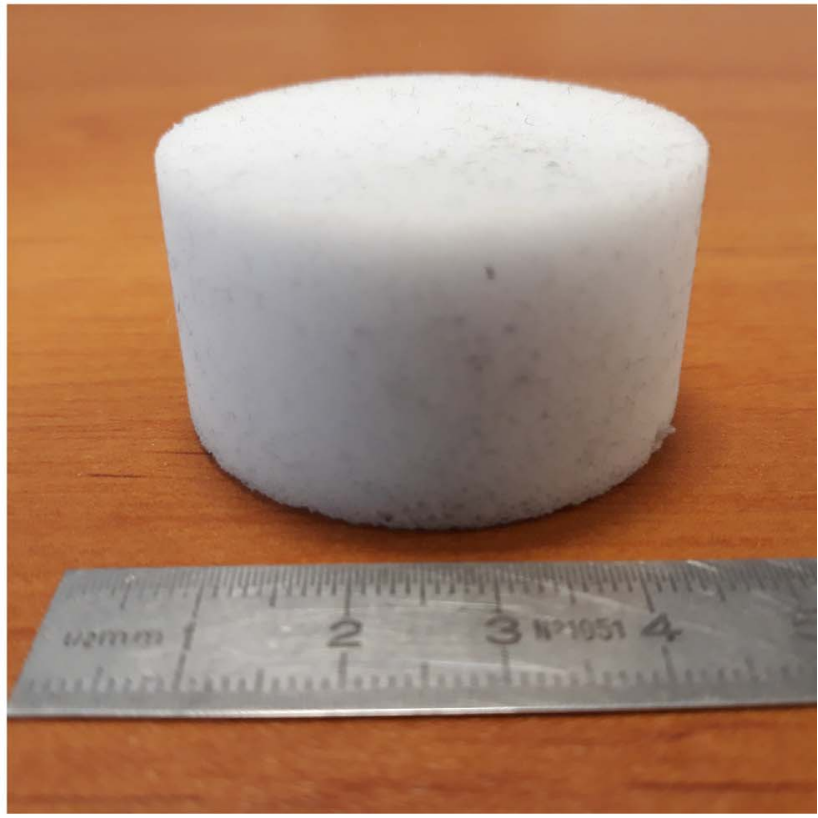

(d) Melamine backing

Figure 7. Photographs of samples of backing and facing media under study.

layers of PEM. This configuration is expected to be more damped meaning that the envelopes should be smoother. This effect is indeed observed and the standard deviation envelope of the experimental results is rather smooth and very well captured by the proposed method for $n=1$. Note that the empirical envelopes might be asymmetrical (for $\theta=60^{\circ}$ for instance) and that this effect is not quite captured by the proposed approach. Nevertheless, the above results support the validity of the proposed approach.

\section{Experimental validation}

Besides the numerical validation of the approach presented in Section 3, a number of experiments presented in the present section allow to assess the capabilities of the proposed method. These experiments use once again two different kinds of facing, one woven and the other nonwoven mimicking their numerical counterpart presented previously, and two different backing media. When needed for the proposed method, the properties of air were chosen the same as in Section 3. Photographs of samples used in the experiments are available in Figure 7.

As a first part of the experimental investigation, the screens and the different backing materials were characterised. The result of this first part is presented in Table 2 in terms of mean value over the measured samples. The characterisation procedure is based on three samples of melamine foam, two samples of glass wool and forty samples of each of the facings. The large number of samples in the last 
Table 2. Material properties from the characterisation process as a first step to the experimental validation (see Sect. 4).

\begin{tabular}{lcccr}
\hline Property $($ unit) & Melamine & Glass wool & Non-woven Film & Woven film \\
\hline Open-porosity $\phi$ & 0.99 & 0.97 & $0.096 \pm 0.01$ & $0.022 \pm 0.003$ \\
Airflow resistivity $\sigma\left(\mathrm{N} \cdot \mathrm{s} \cdot \mathrm{m}^{-4}\right)$ & $11400 \pm 300$ & $76100 \pm 4400$ & $(7189 \pm 1043) \times 10^{2}$ & $(55799 \pm 8305) \times 10^{2}$ \\
Tortuosity $\alpha_{\infty}$ & $1.03 \pm 0.02$ & $1.5 \pm 0.55$ & $1.07 \pm 0.01$ & $46 \pm 3$ \\
Thermal CL $\Lambda^{\prime}(\mu \mathrm{m})$ & $155 \pm 66$ & $64 \pm 30$ & $46 \pm 3$ & $35 \pm 3$ \\
Viscous CL $\Lambda(\mu \mathrm{m})$ & $91 \pm 18$ & $30 \pm 17$ & $427 \pm 14$ & $35 \pm 3$ \\
Density $\rho_{1}\left(\mathrm{~kg} \cdot \mathrm{m}^{-3}\right)$ & $9.2 \pm 0.4$ & $94.1 \pm 2.7$ & $0.59 \pm 0.01$ & $1243.5 \pm 27.3$ \\
Thickness $d(\mathrm{~mm})$ & $24.2 \pm 0.3$ & $48.9 \pm \pm 0.1$ & 0.369 \\
\hline
\end{tabular}

CL stands for characteristic length.

a.

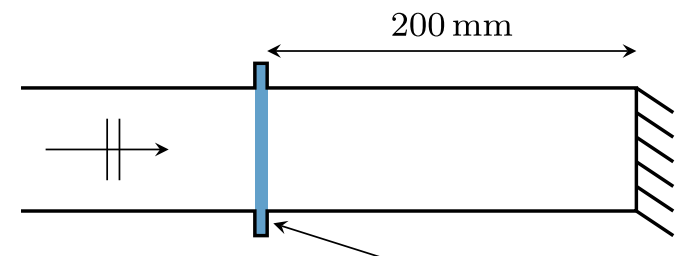

b.

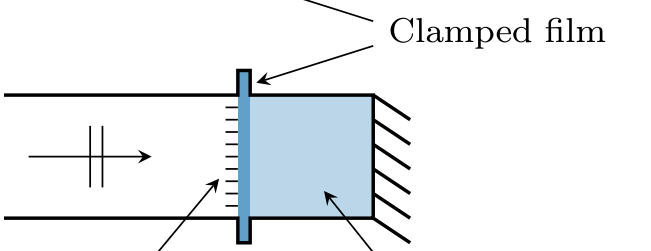

c.

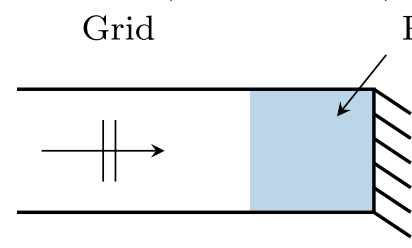

Figure 8. Schematics of the different measurement set-ups. The incident wave is a plane wave, travelling normal to the sample's surface. A three-microphones method is used, one being mounted through the rigid backing, the two others on the side wall between the source and the sample.

case was chosen in order to have statistically significant data.

A second part of the experiments consists in using an impedance tube to measure the response of twenty samples of each film set on one of the samples of melamine and one of the samples of glass wool. This process once again allows to obtain meaningful statistical indicators regarding the response of each system.

The remainder of this section comprises a note on the measurement rig and the post-processing followed by a section of results where the proposed approached is leveraged to predict envelope curves.

The measurements used in the present paper are released online and free to download for further examination or use [19].

\subsection{Measurement rig \& post-processing}

Both for the characterisation of all samples and for the actual measurement of the response of the studied filmbacking configurations, an impedance tube of diameter

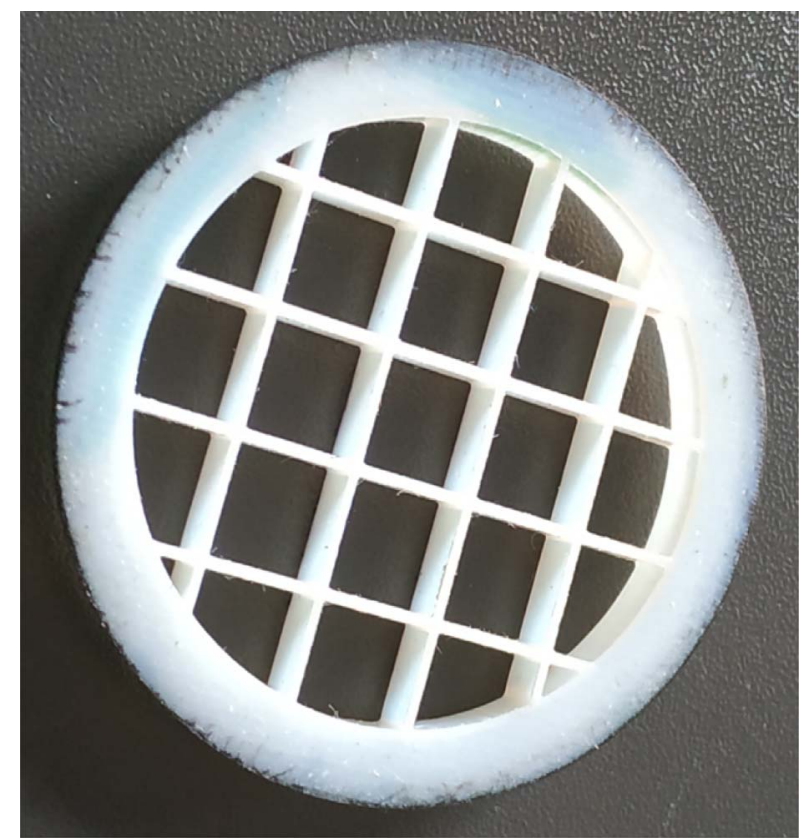

Figure 9. Plastic grid positioned inside the tube to prevent the facings from bending. It adds a $0.89 \mathrm{~mm}$ shift due to its shoulder.

$44.44 \mathrm{~mm}$ was used together with a dedicated commercial software [20]. All measurements use a three-microphones procedure [21, 22] and span over the frequency band 10-4250 Hz. A schematic of the measurement rig is presented on Figure 8. The tube is split in two parts to allow for sample insertion and when needed, the film samples are clamped in the seam. Note that these are thin enough not to create leakage.

Note that the tube is split in two parts which overlap when assembled. If the tube's inner diameter is not impacted by this design, it provides a slight seam located at the beginning of the sample section which can be used for clamping the film samples.

Characterising the samples is entirely based on impedance tube measurements, following procedures described in the literature for facings [13] and backings [23, 24]. In the first case, a $200 \mathrm{~mm}$ plenum is formed behind the screen sample by moving a piston located at the end of the tube. For characterising the backing samples, the piston is adjusted to their length such that the surface of each 

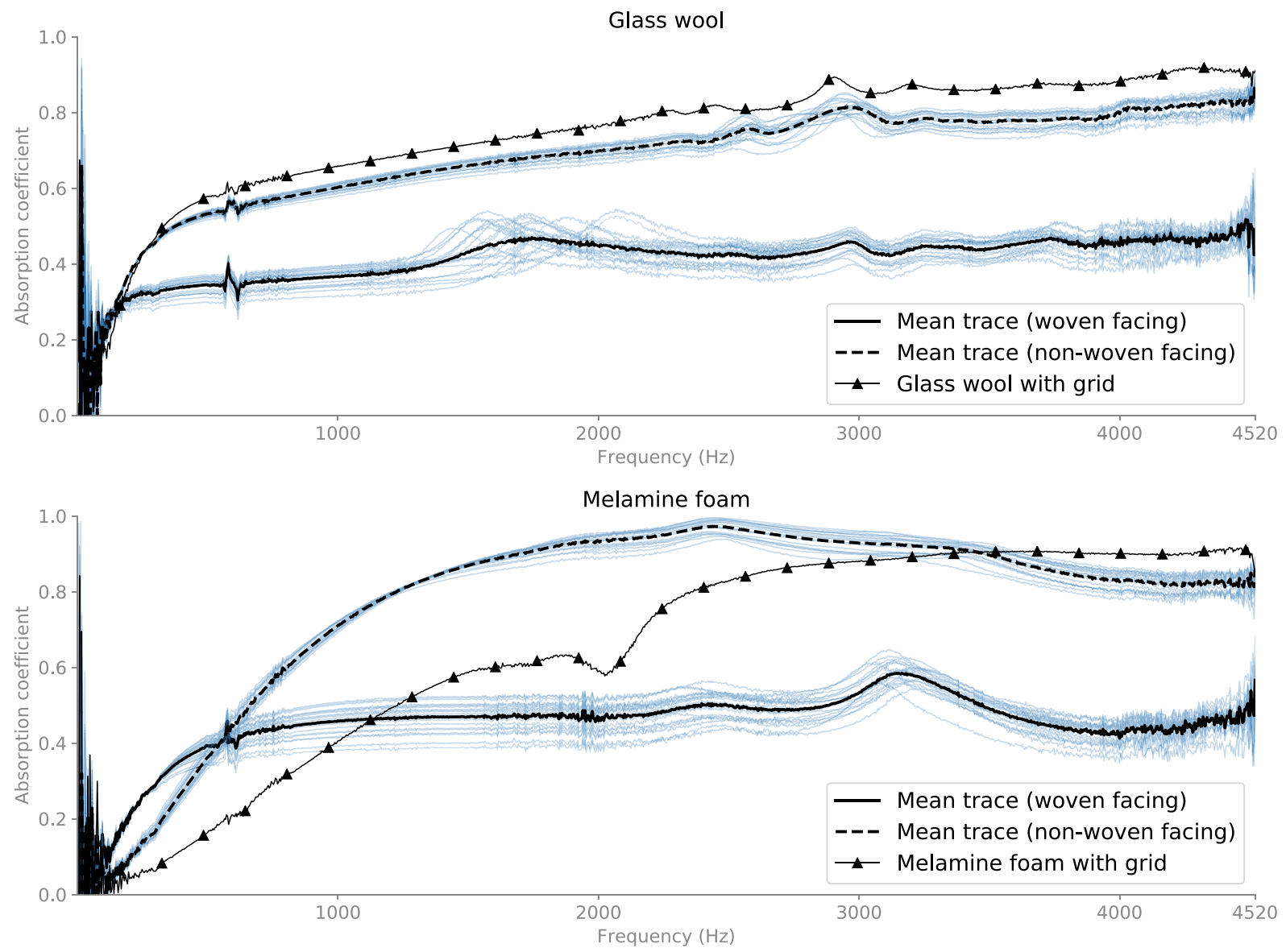

Figure 10. Measured absorption coefficients for the glass wool (top) and melamine (bottom) stacked with each facing (average with woven in solid black line and with the non-woven one in dashed lines) and with only the grid (triangle $\boldsymbol{\Delta}$ markers). Thin blue lines are the traces associated to the different film samples.

sample sits flush with the tube split line (i.e., exactly at the position of the seam). For the two films, the characterisation is performed using 40 samples; for the backings only 3 samples of melamine and 2 samples samples of glass wool were used. The results are gathered in Table 2 .

When measuring the response of the multi-layered systems, in the second part of the experiments, a plastic grid shown in Figure 9 was inserted inside the tube to prevent the facing from bending. This grid has a known thickness of $0.89 \mathrm{~mm}$ which could be compensated for to achieve better accuracy although this was not considered to be necessary here. In an attempt to reduce most of the sources of variation except for these linked to the tested films, the same sample of backing material is used to measure the response with the 20 samples of film. To make this possible, the tested facings are not glued to the backings but simply clamped and mounted tight between the grid and the backing sample. This technique gives satisfying results while being very flexible.

Figure 10 show the absorption coefficient for the two considered backing (melamine foam and glass wool) with the upstream grid as well as the absorption coefficients for each of the 20 samples of film and each backing. On this figure, the influence of the film is clearly visible. Analysing these results, it appears that the variability of the parameters of the film leads to a slight spreading of the responses with occasional features distributed over the spectrum. A glitch is seen around $600 \mathrm{~Hz}$ and is present on all measurements, it is linked to the measurement rig and will not be discussed any further.

To better understand the origin of the observed variations, Figure 11 show the range of variation of the physical parameters for both films in terms of percentage of deviation with respect to the average value. In this figure, it is seen that $\sigma$ is the most varying parameter for both screens, closely followed by the porosity $\phi$ and the two characteristic lengths $\Lambda$ and $\Lambda^{\prime}$. It is known that the airflow resistivity, together with the thickness $d$, is of prime importance as it plays a prominent role in the airflow resistance. Even though the thickness does not vary much amongst the samples considered here, it has been observed to have high deviations for other facings and might sometimes have an important impact.

\subsection{Generating envelopes for experimental data}

In this subsection the envelope response is observed and two techniques to reconstruct these are compared. 


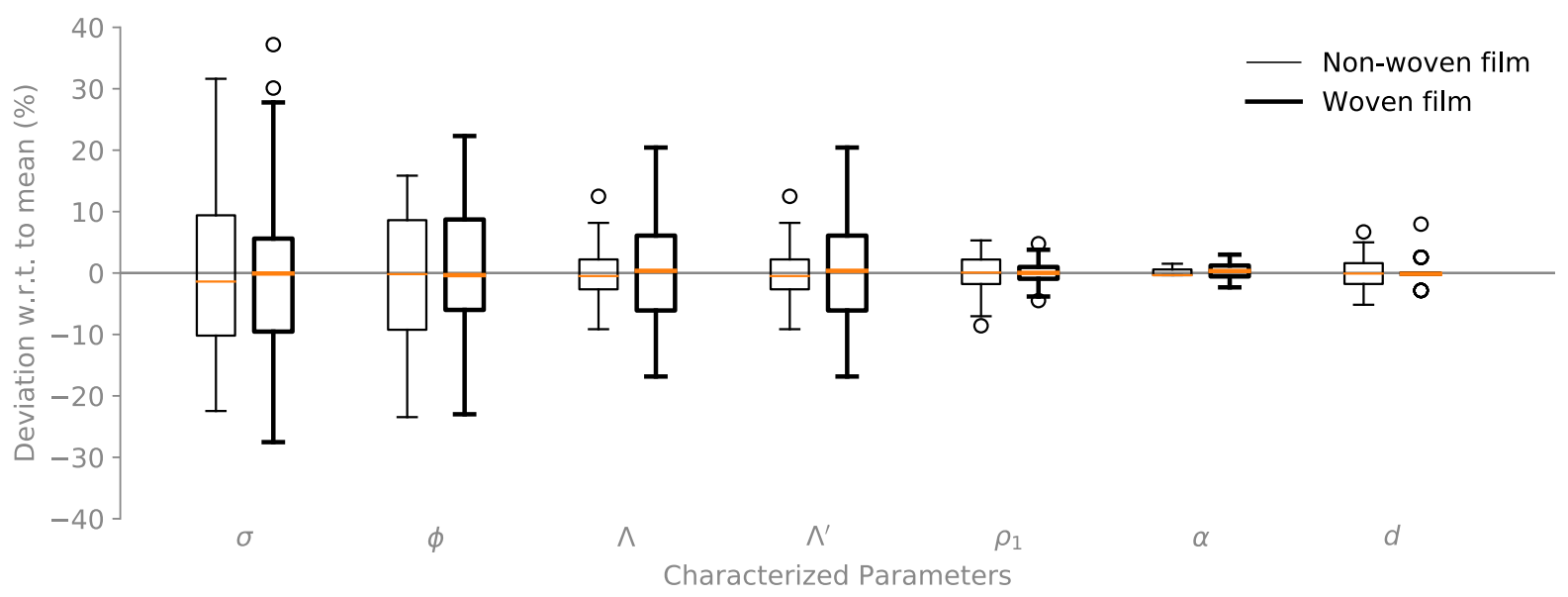

Figure 11. Spread of the JCA parameters and thickness for both facings. The orange line marks the median, outliers are represented with circles and the box spans between the first and third quartiles. Thin lines correspond to the non-woven facing, thick ones to the woven film.
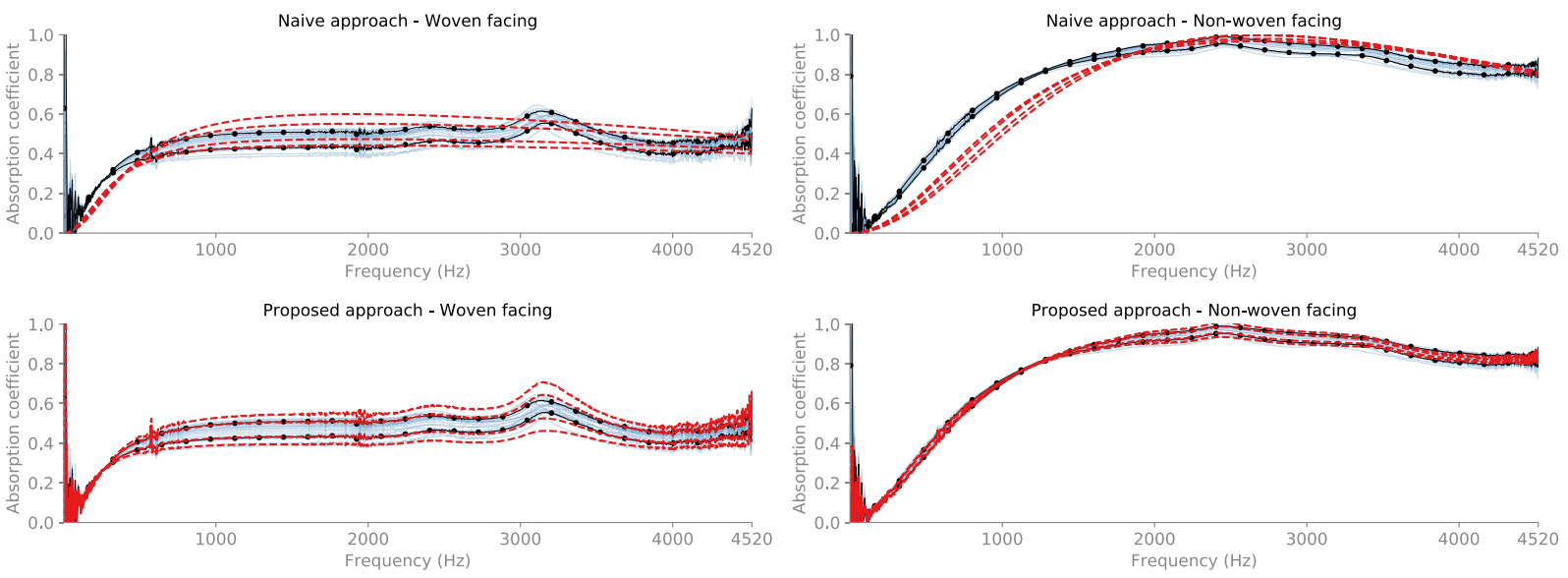

Figure 12. Case of a melamine backing. Comparison of envelopes (in red) annotated on the mean response as proposed in Section 2.3 (bottom) and envelopes generated using the JCA characterisation of the backing and the film (top). For a woven film (left) and a nonwoven (right). The blue light traces are sample responses and the black curve with bullets $\bullet$ is the standard deviation envelope.

The proposed approach is compared to a simplified evaluation based on the mean values and standard deviations of the parameters of the thin facings as well as the backing parameters to compute upper- and lower bound responses. These responses are computed by using the average value of all parameters except the supposedly varying one (for instance $\sigma$ ) for which $n$ times the standard deviation is added/subtracted to mean value.

The other approach is the one described in Section 2 where statistical information on the facing parameter is embedded in an annotated envelope. When needed, the backing impedance $Z_{B}$ is computed using the average value of the parameters. All envelopes in this section are computed with the proposed method for $n=1$ or $n=2$. On Figure 12, the cases with a melamine backing is presented, on Figure 13 the one with a glass wool backing is shown.
Note that in these figures, both films are considered for both approaches.

From these figures, it is clear that the simplified approach is rarely a good approximation of the actual envelope, particularly at low frequencies. On the top rows of both Figures 12 and 13, one easily notices the discrepancies introduced by considering a JCA model both for the backing and for the film. Indeed, even though this hypothesis is reasonable for the film thanks to its very specific effect and thinness, it is a strong approximation for the poro-elastic backing in which solid-borne waves also propagate.

On the other hand the proposed approach closely follows the empirical envelope particularly the predicted envelope for $n=1$ which almost exactly follows the standard deviation envelope. Some discrepancies are observed 

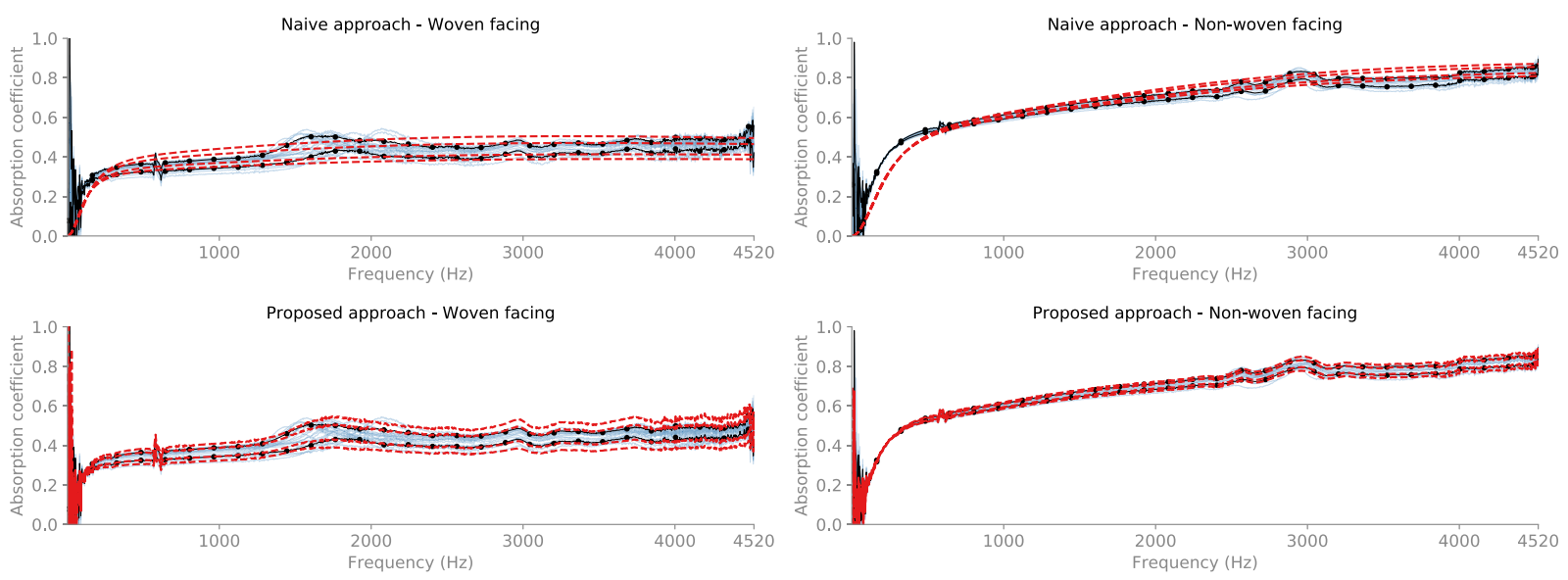

Figure 13. Case of a glass wool backing. Comparison of envelopes (in red) annotated on the mean response as proposed in Section 2.3 (bottom) and envelopes generated using the JCA characterisation of the backing and the film (top). For a woven film (left) and a nonwoven (right). The blue light traces are sample responses and the black curve with bullets $\bullet$ is the standard deviation envelope.

around $1750 \mathrm{~Hz}$ on Figure 13 with a woven fabric, which might be linked to the fact that the surface of the glasswool sample is not perfectly flat and that the woven film is rather soft. Around this frequency, the proposed approach traces a plausible envelope but does not account for the existing resonance.

\section{Conclusion}

In the present contribution, a method to generate envelopes responses for multi-layered systems including a film with uncertain parameters is introduced. The proposed method uses a set of Taylor expansions to introduce a way to separate the contributions of uncertainties from the average trend. The said method is both suitable for standalone computations of envelope responses or as a tool to compute envelopes from a single response from the system and the parameters of the facing.

The method is tested both on simulated cases with materials and configurations sourced from the literature and on actual experiments. In both cases, the method is shown to give good results with predicted envelopes having similar trends and amplitudes as the ones based on measurements. The proposed solution is fitting the measured reference better than a simplified approach based on the bounds of a given variation interval of the parameters.

\section{Acknowledgments}

The authors would like to express their gratitude towards one of the reviewers suggesting the use of the total differential in equation (7).

\section{Conflict of interest}

Author declared no conflict of interests.

\section{References}

1. J.-F. Allard, N. Atalla: Propagation of Sound in Porous Media: Modelling Sound Absorbing Materials. 2nd edn., Wiley, Hoboken, NJ, 2009.

2. T. Weisser, J.-P. Groby, O. Dazel, F. Gaultier, E. Deckers, S. Futatsugi, L. Monteiro: Acoustic behavior of a rigidly backed poroelastic layer with periodic resonant inclusions by a multiple scattering approach. The Journal of the Acoustical Society of America 139 (2016) 617-629. https://doi.org/ 10.1121/1.4940669.

3. N. Jiménez, V. Romero-García, V. Pagneux, J.-P. Groby: Rainbow-trapping absorbers: Broadband, perfect and asymmetric sound absorption by subwavelength panels for transmission problems. Scientific Reports 7 (2017) 13595. https://doi.org/10.1038/s41598-017-13706-4.

4. C. Boutin: Acoustics of porous media with inner resonators. The Journal of the Acoustical Society of America 134 (2013) 4717-4729. https://doi.org/10.1121/1.4824965.

5. M.E. Beutel, C. Jünger, E.M. Klein, P. Wild, K. Lackner, M. Blettner, H. Binder, M. Michal, J. Wiltink, E. Brähler, T. Münzel: Noise annoyance is associated with depression and anxiety in the general population - The contribution of aircraft noise. PLoS One 11 (2016) e0155357. https://doi. org/10.1371/journal.pone.0155357.

6. The European Parliament: Regulation (EU) No 540/2014 of the European Parliament and of the Council of 16 April 2014 on the sound level of motor vehicles and of replacement silencing systems, and amending Directive 2007/46/EC and repealing Directive $70 / 157 /$ EEC. Official Journal of the European Communities L158 (2014) 131-195.

7. W. Babisch: The noise/stress concept, risk assessment and research needs. Noise \& Health 4 (2002) 1-11.

8. F. Chevillotte: Controlling sound absorption by an upstream resistive layer. Applied Acoustics 73 (2012) 56-60. https:// doi.org/10.1016/j.apacoust.2011.07.005.

9. S. Sugie, J. Yoshimura, H. Ogawa: Absorption characteristics of fibrous material covered with perforated facing and film. Acoustical Science and Technology 27 (2006) 87-96.

10. N. Atalla, F. Sgard: Modeling of perforated plates and screens using rigid frame porous models. Journal of Sound and Vibration 303 (2007) 195-208. https://doi.org/10.1016/ j.jsv.2007.01.012. 
11. M. Gaborit, O. Dazel, P. Göransson: A simplified model for thin acoustic screens. The Journal of the Acoustical Society of America 144 (2018) EL76-EL81. https://doi.org/10.1121/ 1.5047929 .

12. A.D. Pierce: Acoustics: An Introduction to its Physical Principles and Applications. 1989th ed., Acoustical Society of America, Woodbury, NY, 1989.

13. L. Jaouen, F.-X. Bécot: Acoustical characterization of perforated facings. The Journal of the Acoustical Society of America 129 (2011) 1400-1406. https://doi.org/10.1121/ 1.3552887.

14. O. Dazel, B. Brouard, C. Depollier, S. Griffiths: An alternative Biot's displacement formulation for porous materials. JASA 121 (2007) 3509. https://doi.org/10.1121/ 1.2734482 .

15. Y. Champoux, J.-F. Allard: Dynamic tortuosity and bulk modulus in air-saturated porous media. Journal of Applied Physics 70 (1991) 1975. https://doi.org/10.1063/ 1.349482 .

16. D.L. Johnson, J. Koplik, R. Dashen: Theory of dynamic permeability and tortuosity in fluid-saturated porous media. Journal of Fluid Mechanics 176 (1987) 379-402.

17. ISO: ISO 9053-1 - Acoustics - Determination of Airflow Resistance - Static Airflow Method. 2018.
18. ISO: ISO 10534-2 Acoustics - Determination of Sound Absorption Coefficient and Impedance in Impedance Tubes Part 2: Transfer-Function Method. 1998.

19. M. Gaborit, L. Jaouen, O. Dazel, P. Göransson: Statistical characterisation and response of acoustic screens and twolayers systems (data set). Zenodo. 2019. https://doi.org/ 10.5281 /zenodo.3358921.

20. Matelys: TubeCell. 2019.

21. T. Iwase, Y. Izumi: A new sound tube measuring method for propagation constant in porous material - Method without any air space at the back of test material, Journal of the Acoustical Society of Japan 52 (1996) 411-419.

22. T. Iwase, Y. Izumi, R. Kawabata: A new measuring method for sound propagation constant by using sound tube without any air spaces back of a test material, in INTER-NOISE and NOISE-CON Congress and Conference Proceedings Vol. 1998, INCE. 1998. pp. 1265-1268.

23. R. Panneton, X. Olny: Acoustical determination of the parameters governing viscous dissipation in porous media. The Journal of the Acoustical Society of America 119 (2006) 2027. https://doi.org/10.1121/1.2169923.

24. X. Olny, R. Panneton: Acoustical determination of the parameters governing thermal dissipation in porous media. The Journal of the Acoustical Society of America 123 (2008) 814-824. https://doi.org/10.1121/1.2828066.

Cite this article as: Gaborit M, Dazel O, Göransson P \& Jaouen L. 2020. Generation of uncertainty envelopes for thin acoustic screens with uncertain parameters. Acta Acustica, 4, 8. 